\title{
Basilar Artery Hypoplasia with Embryonic Origin of Anterior Cerebral Arteries: A Radiological Gamut
}

Razak MK and Meena GL*

Department of Radiodiagnosis, Sardar Patel Medical College, Bikaner, Rajasthan, India

\begin{abstract}
Aims and Objectives: This article aims to determine the most frequent cause of basilar hypoplasia and evaluate the potential impact of associated pathological findings simultaneously.

Material and Methods: We have retrospectively reviewed the patients in our archive with basilar hypoplasia and embryonic origin of ACPs. These patients have been studied using 3D TOF with 3D-VR reconstruction. The studies have been carried out using MRI of 1.5 and $3 \mathrm{~T}$. Several of them have also undergone cerebral angiography by digital subtraction.

Results: The most frequent cause of basilar hypoplasia has been associated with the embryonic origin of both bilateral ACPs with 27 cases. In 3 cases, the basilar artery hypoplasia has been associated with persistent trigeminal artery. In 25 patients studied the basilar hypoplasia was an incidental finding in the context of a routine MRI study including angio MRI sequences. Of the patients studied only 5 had allegedly clinical history that could be related to hypoplastic basilar artery.

Conclusion: Hypoplasia of basilar artery is a related variant, in most cases, with both embryonic origins of ACPs. In most of the patients studied, this was an incidental finding. However the said variant may be a predisposing factor to cause any grade of vascular insufficiency in the territory of vertebro-basilar artery. With respect to the aneurysm, associated described persistent trigeminal artery is thought likely may have a causal relationship with this variant.
\end{abstract}

Keywords: Cerebral; Arteries; Imaging; CT; Angio

\section{Introduction}

Before we begin to describe the article, it is imperative to elucidate the relevant anatomy for a better understanding of the topic (Figure 1) [1].

\section{Embryonic origin of the posterior cerebral arteries (ACPs)}

It occurs when unilateral or bilateral regression of one of the fetal posterior cerebral arteries does not occur, embryological origin being conserved in the ICA. The P1 segment is hypoplasic or absent [2].

\section{Hypoplasia of the basilar artery (AB)}

The definition of $\mathrm{AB}$ hypoplasia is not well established in the literature. We consider artery hypoplasia when the diameter of said

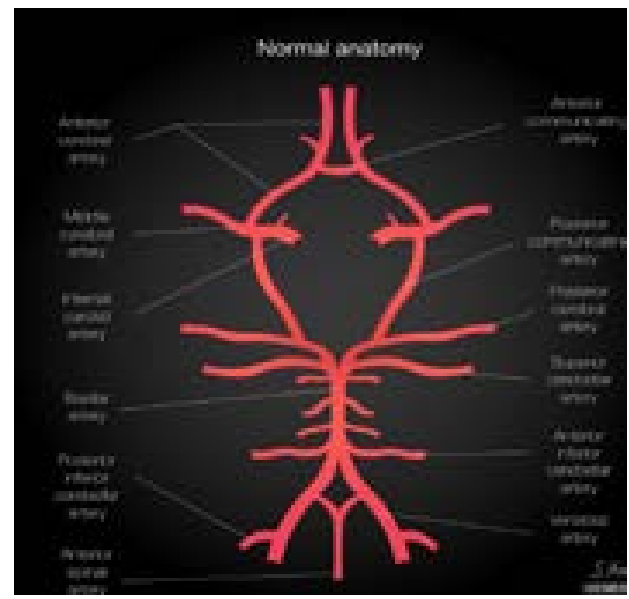

Figure 1: A pictographic representation of the circle of Willis. vessel (caliber) in its distal third is less than the diameter of at least one of the ACP. In most cases it corresponds to a diameter less than $2 \mathrm{~mm}$.

\section{Trigeminal artery}

Embryological connection between the cavernous portion of the carotid arteries and the longitudinal arteries will later fuse to form the trunk of the basilar artery [3].

\section{Classic Willis polygon}

Defined as the cerebral vascular ring conformed by the following vascular structures (Figures 2 and 3) [4]:

- 2 internal carotid arteries (ACA).

- 2 segments A1 of the anterior cerebral arteries (ACAs).

- 1 anterior communicating artery (ACoA).

- 2 horizontal segments of both posterior cerebral arteries (ACAs).

- 2 posterior communicating arteries (ACoA).

- 1 basilar artery $(\mathrm{AB})$.

*Corresponding author: Meena GL, Senior Professor, H.O.D, Department of Radiodiagnosis, S.P. Medical College, Bikaner, Rajasthan-334001, India, Tel: 09414042 908; E-mail: meenabkn@yahoo.co.in

Received December 06, 2017; Accepted December 08, 2017; Published December 11, 2017

Citation: Razak MK, Meena GL (2017) Basilar Artery Hypoplasia with Embryonic Origin of Anterior Cerebral Arteries: A Radiological Gamut. Human Genet Embryol 7: 144. doi:10.4172/2161-0436.1000144

Copyright: (c) 2017 Razak MK, et al. This is an open-access article distributed under the terms of the Creative Commons Attribution License, which permits unrestricted use, distribution, and reproduction in any medium, provided the original author and source are credited. 


\section{Material and Methods}

We have retrospectively reviewed the patients in our archive with basilar hypoplasia and embryonic origin of ACPs (Figures 4-10).

These patients have been studied using 3D TOF (time of flight) with 3D-VR (volume reconstruction). The studies have been carried out using MRI of 1.5 and 3T. Several of them have also undergone cerebral angiography by Digital Subtraction (DSA).

\section{Results and Discussion}

\section{Incidence of basilar artery hypoplasia (AB)}

The hypoplasia of basilar artery is a pathological activity, observed with some frequency in the MRI and CT scans most often incidentally.

The most common presentation of hypoplasia of the basilar artery is associated with the persistence of embryonic origin of the posterior

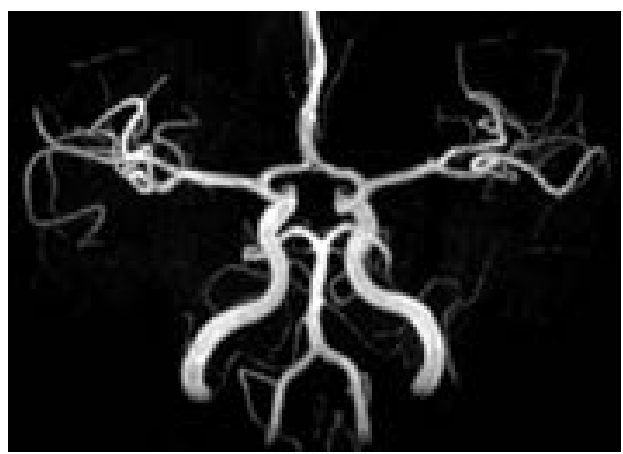

Figure 2: Circle of Willis classic: Angio RM 3D MIP (maximum intensity projection).

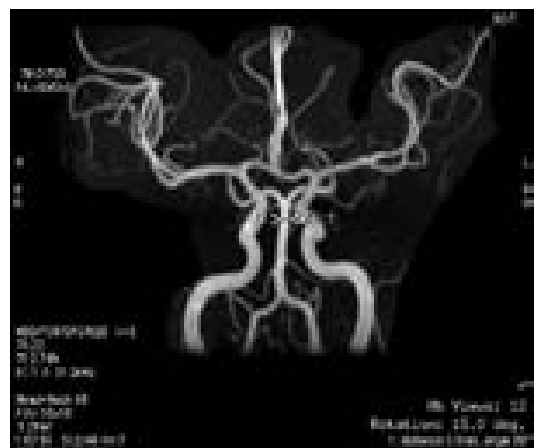

Figure 3: Basilar artery $(A B)$ normal diameter $(3.1 \mathrm{~mm})$.

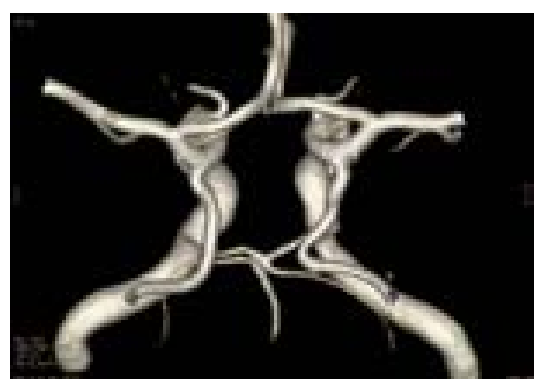

Figure 4: Case of embryonic origin of both posterior cerebral arteries (ACAs) is displayed. Angio RM. 3D VR.

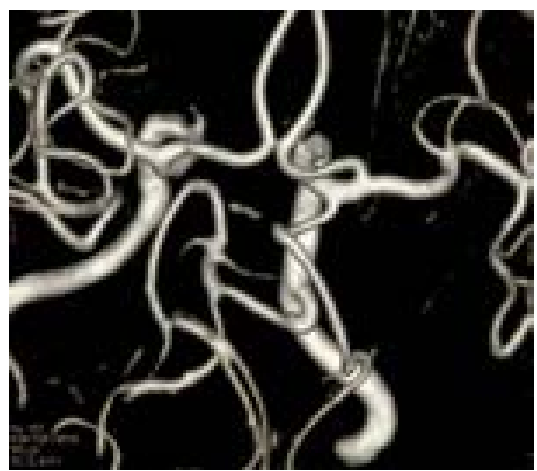

Figure 5: Trigeminal artery persistent left. ACoA hypoplasia of the left and right embryonic origin of ACA.

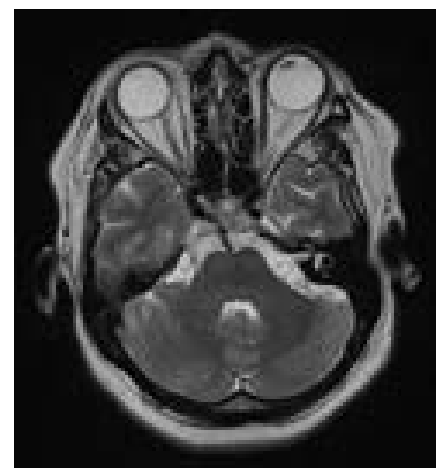

Figure 6: Sequence axial T2: Hypoplasia basilar artery (punctate image) and trigeminal largest artery.

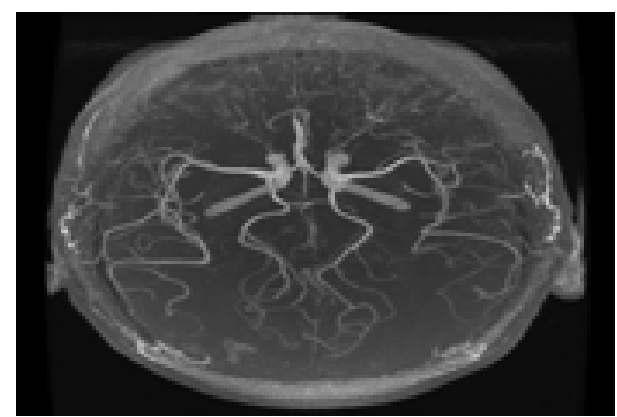

Figure 7: Ex. Case 1: Hypoplasia AB $(1.4 \mathrm{~mm})$ associated with both embryonic ACAs. Below: Angio RM (MIP).

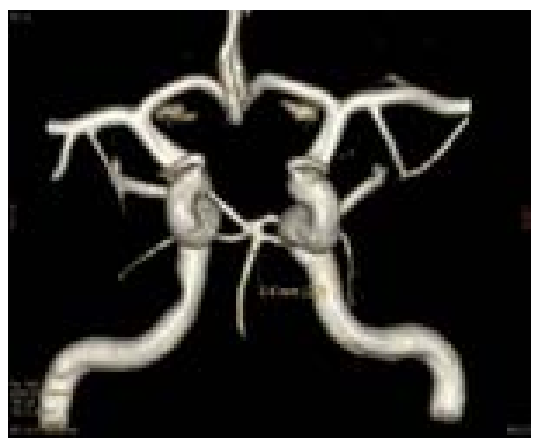

Figure 8: Ex. Case 1: Hypoplasia $A B(1.4 \mathrm{~mm})$ associated with embryonic origin of the two ACAs: 3D Reconstruction VR TOF. 


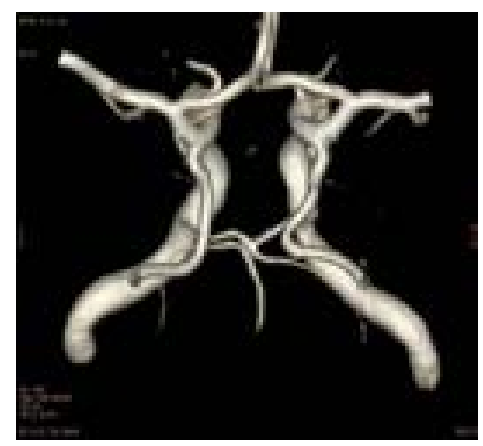

Figure 9: Ex. Case 1: Basilar artery hypoplasia associated with both embryonic origin of ACAs: Right: VR 3D TOF reconstruction.

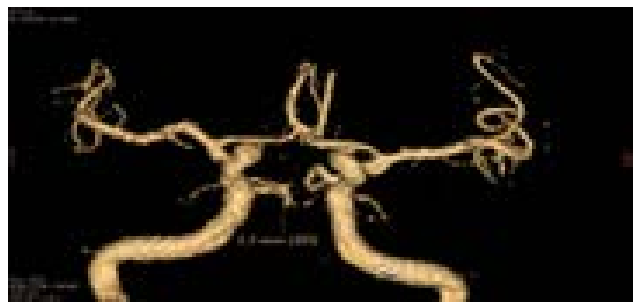

Figure 10: Ex. Case 2: Marked hypoplasia $A B(1.3 \mathrm{~mm})$ associated with both embryonic origin of ACAs.. an accessory branch of the ACA is observed originating from the ACoA. TOF $3 \mathrm{D}$ VR reconstruction.

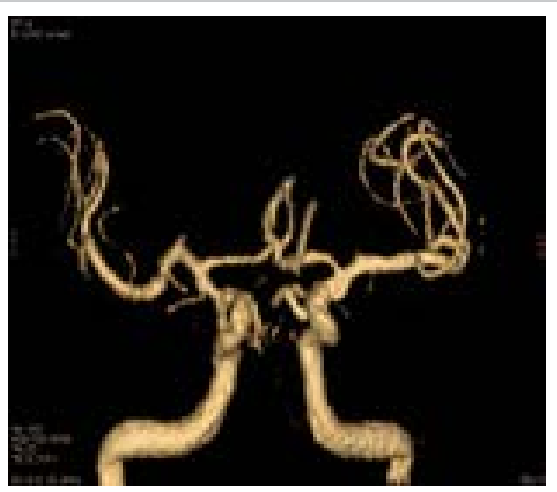

Figure 11: Ex. Case 2: Marked hypoplasia to basilar (partially displayed) associated with both embryonic origin of ACPs. An accessory branch of the $A C A$ is observed originating from the ACOA. TOF 3D VR reconstruction.

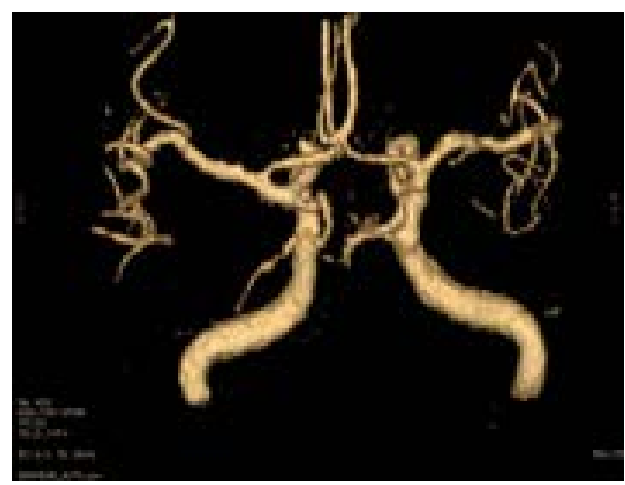

Figure 12: Ex. Case 2: Marked hypoplasia associated with both embryonic origin of ACAs. An accessory branch of ACA is also observed originating from the ACoA.

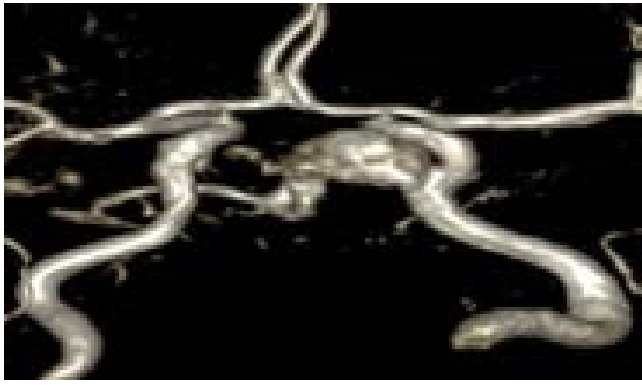

Figure 13: Ex. Case 3: Persistent trigeminal artery (tau sign) with aneurysmal dilatation in origin. TOF 3D image.

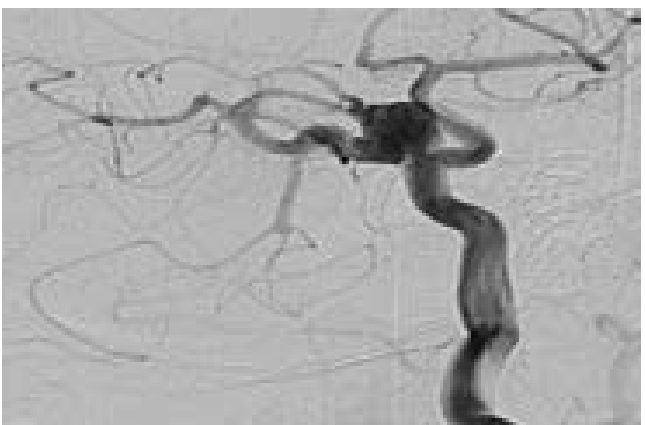

Figure 14: Ex. Case 3: DSA shows a persistent trigeminal artery with aneurysmal dilatation in origin.

cerebral artery (PCA) with hypoplasia segment P1, objectifying up to $8 \%$ of cases bilaterally.

We have performed a retrospective study of such vascular variant. Here are the images of several cases in our series of 30 patients studied by MRI and Angio RM.

- The most frequent cause of hypoplastic basilar artery has been associated with the embryonic origin of both bilateral ACA's with 27 cases (Figures 11 and 12).

- In 3 cases, the hypoplasia basilar artery has been associated with persistent trigeminal artery (Figures 13 and 14).

- In 25 patients studied the basilar hypoplasia was an incidental finding in the context of a routine MRI study including Angio MRI sequences.

- Of the patients studied only 5 had allegedly clinic history that could be related to the hypoplastic basilar artery.

\section{Conclusion}

Hypoplasia of basilar artery is a related variant, in most cases, with both embryonic origins of ACPs. In most of the patients studied, this was an incidental finding [5]. However the said variant may be a predisposing factor to cause any grade of vascular insufficiency in the territory of vertebro-basilar artery. With respect to the aneurysm, associated described persistent trigeminal artery is thought likely may have a causal relationship with this variant [6].

\section{References}

1. Azab W, Delashaw J, Mohammed M (2012) Persistent primitive trigeminal artery: A review. Turk Neurosurg 22: 399-406.

2. Osborn AG (2012) Osborn's Brain: Imaging, pathology and anatomy. Las Edition. 
Citation: Razak MK, Meena GL (2017) Basilar Artery Hypoplasia with Embryonic Origin of Anterior Cerebral Arteries: A Radiological Gamut. Human Genet Embryol 7: 144. doi:10.4172/2161-0436.1000144

3. Dimmick SJ, Faulder KC (2009) Normal variants of the cerebral circulation at multi-detector CT angiography. Radiographics 29: 1027-1043.

4. Meckel S, Spittau B, McAuliffe W (2013) The persistent trigeminal artery: development, imaging anatomy, variants and associated vascular pathologies. Neuroradiology 55:5-16.
5. O'uchi E, O'uchi T (2010) Persistent primitive trigeminal arteries (PTA) and its variant (PTAV): Analysis of 103 cases detected in 16,415 cases of MRA over 3 years. Neuroradiology 52: 1111-1119.

6. Boyko OB, Curnes JT, Blatter DD, Parker DL (1996) MRI of basilar artery hypoplasia associated with persistent primitive trigeminal artery. Neuroradiology 38: 11-14. 